\title{
Development and Evaluation of a Decision Aid for BRCA Carriers with Breast Cancer
}

\author{
Julie O. Culver ${ }^{1}$, Deborah J. MacDonald ${ }^{1}$, Andrea A. Thornton ${ }^{2}$, Sharon R. Sand ${ }^{1}$, Marcia \\ Grant $^{3}$, Deborah J. Bowen ${ }^{4}$, Harry Burke ${ }^{5}$, Nellie Garcia ${ }^{6}$, Kelly A. Metcalfe ${ }^{7}$, and Jeffrey N. \\ Weitzel $^{1}$ \\ ${ }^{1}$ Division of Clinical Cancer Genetics, Department of Population Sciences, City of Hope \\ Comprehensive Cancer Center, Duarte, CA
}

2Division of Behavioral Oncology, Department of Population Sciences, \& Division of Psychology, Department of Supportive Care Medicine, City of Hope Comprehensive Cancer Center, Duarte, CA

${ }^{3}$ Division of Nursing Research and Education, Department of Population Sciences, City of Hope Comprehensive Cancer Center, Duarte, CA

${ }^{4}$ Boston University, Department of Social and Behavioral Sciences, Boston, MA

${ }^{5}$ George Washington University School of Medicine, Department of Medicine, Biochemistry and Molecular Biology, Washington, DC

${ }^{6}$ Department of Clinical Social Work, Sheri \& Les Biller Patient and Family Resource Center, City of Hope Comprehensive Cancer Center, Duarte, CA

7 University of Toronto, Women's College Research Institute, Toronto, Ontario, Canada

\section{Abstract}

$B R C A+$ breast cancer patients face high risk for a second breast cancer and ovarian cancer. Helping these women decide among risk-reducing options requires effectively conveying complex, emotionally-laden, information. To support their decision-making needs, we developed a web-based decision aid (DA) as an adjunct to genetic counseling. Phase 1 used focus groups to determine decision-making needs. These findings and the Ottawa Decision Support Framework guided the DA development. Phase 2 involved nine focus groups of four stakeholder types (BRCA + breast cancer patients, breast cancer advocates, and genetics and oncology professionals) to evaluate the DA's decision-making utility, information content, visual display, and implementation. Overall, feedback was very favorable about the DA, especially a values and preferences ranking-exercise and an output page displaying personalized responses. Stakeholders were divided as to whether the DA should be offered at-home versus only in a clinical setting. This well-received DA will be further tested to determine accessibility and effectiveness.

\section{Keywords}

BRCA1; BRCA2; breast cancer; decision aid; decision-making; genetic counseling; mastectomy; oophorectomy

Corresponding author: Julie Culver, MS, CGC Division of Clinical Cancer Genetics City of Hope 1500 East Duarte Road Mod 173 Duarte, CA 91010-3000 626-256-8662 × 7 (phone) 626-930-5495 (fax) jculver@coh.org.

DISCLOSURE OF INTEREST There are no actual or potential conflicts of interest. Authors have full control of primary data and consent to journal review of data if necessary. 


\section{INTRODUCTION}

Breast cancer patients with a $B R C A 1$ or $B R C A 2$ gene mutation $(B R C A+)$ have a $30-60 \%$ risk of developing a second primary breast cancer (Ford et al., 1994; Metcalfe et al., 2004) and an 11-45\% risk for ovarian cancer (Antoniou et al., 2003; Ford et al., 1998; Struewing et al., 1997). These high risks challenge women to consider interventions such as risk-reducing mastectomy, risk-reducing salpingo-oophorectomy, and use of tamoxifen. Risk-reducing mastectomy confers a $>90 \%$ breast cancer risk reduction (Domchek et al., 2010; McDonnell et al., 2001; Rebbeck et al., 2004). Risk-reducing salpingo-oophorectomy provides $>90 \%$ ovarian cancer risk reduction (Hartmann et al., 1999; Kauff et al., 2002; Rebbeck et al., 2004; Rebbeck et al., 2002), substantially lowers breast cancer risk for pre-menopausal women (Kauff et al., 2002; Rebbeck, Kauff, \& Domchek, 2009; Rebbeck et al., 2002), and reduces all-cause and cancer-specific mortality (Domchek et al., 2010). Tamoxifen reduces breast cancer risk in BRCA+ women by about 50\% (King et al., 2001; Narod et al., 2000). Therefore, the National Comprehensive Cancer Network guidelines for female BRCA mutation carriers include consideration of risk-reducing mastectomy and tamoxifen and a recommendation for risk-reducing salpingo-oophorectomy after completion of childbearing (NCCN, 2010). Yet risk-reducing mastectomy and risk-reducing salpingo-oophorectomy are irreversible and women contemplating these surgeries may have significant distress and anxiety, particularly if also making decisions about breast cancer treatment (Croyle, Smith, Botkin, Baty, \& Nash, 1997; Watson et al., 2004).

Decision aids (DAs) have been shown to improve patients' ability to make decisions, including cancer-related decision-making (O'Brien et al., 2009; O'Connor et al., 1999; Waljee, Rogers, \& Alderman, 2007; Whelan et al., 2004). A review of 200 DAs, including 34 randomized controlled trials comparing DAs to usual care found greater knowledge, more realistic expectations, lower decisional conflict, more patient involvement in decisionmaking and fewer undecided patients post-intervention (O'Connor et al., 2004).

Decision aids have been developed for $B R C A+$ women considering preventive and screening options. In 1998, a Dutch team developed a brochure and videotape for $B R C A+$ women $(n=89)$ which was found to help patients feel more informed, more satisfied with the information, and have more accurate risk perceptions (van Roosmalen et al., 2004a). The same team also tested a shared decision making intervention for the same group of $B R C A+$ women which had mixed results; at nine months follow-up, unaffected $B R C A$ carriers in the intervention arm $(n=33)$ had lower decisional uncertainty, higher perceived decisionmaking participation, and stronger decision preferences, while women with $\mathrm{BC}(n=11)$ did not achieve similar benefits (van Roosmalen et al., 2004b). An Australian group developed a tailored booklet with a values clarification exercise for women at genetic risk for ovarian cancer due to a BRCA mutation or Lynch Syndrome. In a randomized trial comparing the tailored decision aid booklet to a general educational pamphlet the researchers found that those receiving a tailored DA ( $n=68)$ demonstrated a significant decrease in decisional conflict and greater increases in knowledge than the control group $(n=63)$ (Tiller et al., 2006).

A paper-based DA for unaffected women with a $B R C A$ mutation led to a significant decline in mean decisional conflict, increase in knowledge scores, and less uncertainty about prophylactic surgery $(n=20)$ (Metcalfe et al., 2007). In a randomized trial of a CD-ROMbased DA for $B R C A+$ women ( $n=214$ ), the DA was effective in helping undecided $B R C A$ carriers reach a management decision, decreased their decisional conflict, and increased their decision satisfaction (Schwartz et al., 2009) as well as reduced cancer-specific and genetic testing-specific distress (Hooker et al., 2010). These data support the use of DAs in 
the $B R C A+$ population to increase satisfaction and knowledge and decrease decisional conflict.

However, none of the existing decision aids are both (1) designed for use with $B R C A+$ women with breast cancer to help make risk-reducing decisions such as risk-reducing mastectomy and risk-reducing salpingo-oophorectomy and (2) available in a format that has been updated with recent medical advances. Women with a new diagnosis of breast cancer must integrate knowledge of their BRCA status into their surgical treatment decisions (Mai, Lagos, Palomares, \& Weitzel, 2008; Schwartz et al., 2004; Weitzel et al., 2003). Women with a past diagnosis of breast cancer may face survivorship issues that may also influence their decision-making, such as menopause, infertility, fear of recurrence, and family distress (Crotser \& Boehmke, 2009; Ferrell, Grant, Funk, Otis-Green, \& Garcia, 1997). Knowledge of one's $B R C A+$ status adds additional burden related to worry about a possible future primary cancer. A DA designed for this population could help address their unique needs.

We report qualitative data gathered from patients, advocates and providers during the development of a web-based DA for women with a new or past diagnosis of breast cancer who receive a $B R C A+$ genetic test result. The DA was designed to be used as an adjunct to genetic counseling. Development of the DA was guided by the Ottawa Decision Support Framework, a conceptual framework advocating clear presentation of risk information and values clarification (O'Connor et al., 1998). Our objectives were to: 1) gain a better understanding of the unique decision-making needs of $B R C A+$ women with breast cancer; and, 2) develop an effective, easily accessible and updateable DA for this high-risk population.

\section{METHODS}

\section{Design}

A focus group strategy was chosen as the optimal means of eliciting data to guide development of the DA. Focus groups are especially useful in the early stages of research on unexplored topics, as they allow for a fuller understanding of the decision-making process, and facilitate participant interaction (Krueger \& Casey, 2000). Focus group data were collected at two points during the DA development. First, two focus groups of BRCA+ women with a history of breast cancer were held to determine their decision-making support needs related to cancer risk-reduction (Phase 1). Findings were then used to develop the web-based DA. Next, nine additional focus groups with former breast cancer patients, advocates, and health professionals were conducted to evaluate the DA (Phase 2). The study was approved by the City of Hope (COH) Institutional Review Board.

\section{Phase 1 Focus Groups}

Participants-Eligibility criteria included being female, English-speaking, age $\geq 21$ years, $B R C A+$, history of stage 0-IIIa breast cancer, 6 months past initial genetic counseling and previously enrolled in the $\mathrm{COH}$ Clinical Cancer Genetics Hereditary Cancer Registry allowing re-contacting for research studies. Eligible women $(N=120)$ were mailed a study invitation packet including a pre-addressed/stamped response card. After two weeks, nonrespondents received a telephone call. Participants were offered a $\$ 25$ gas card for completing the focus group.

Procedures-The Phase 1 focus groups held in 2006 at COH were led by a trained facilitator and co-facilitator using a structured interview guide and established focus group methods (Krueger \& Casey, 2000). Participants provided demographic information (Table I) and written informed consent to participate. Participants were asked questions about the 
influence of having a $B R C A$ mutation on their surgical and other risk-reduction decisions, the factors they considered in their decision-making, and the process by which their decisions were reached.

Data Analysis-Qualitative thematic analysis procedures guided data analysis (Braun \& Clarke, 2006; Hsieh \& Shannon, 2005; Krueger \& Casey, 2000). The audio-tapes were transcribed verbatim and then checked for accuracy by a study team member present at the focus groups. To provide a systematic means of organizing the data into central themes, investigators developed a coding list using categories derived from the questions asked in the interview guide. Text that did not fit the categories were assigned a new category (Hsieh \& Shannon, 2005). To establish inter-rater reliability, two investigators coded each transcript independently (Neuendorf, 2002). The few minor differences of opinion were discussed between the reviewers until consensus was reached. To facilitate coding, the qualitative data analysis program ATLAS.ti (Muhr, 2005) was used. Subsequently, each category of data was analyzed by two investigators to identify key opinions, ideas, and themes; collect quotes; and identify points of agreement and disagreement between participants (Braun \& Clarke, 2006; Hsieh \& Shannon, 2005; Krueger \& Casey, 2000).

\section{Development of the Decision Aid}

Conceptual Framework-The DA was guided by the Ottawa Decision Support Framework (O'Connor et al., 1998). This is an evidence-based, transdisciplinary framework for making health decisions stimulated by new health circumstances that require careful deliberation because of the uncertainties or value-sensitive nature of the benefits and risks of treatment. Also this framework is particularly helpful for treatment decisions requiring significant effort during the deliberation phase. The framework has three elements: assessing the needs or determinants of the decision, providing decision support, and evaluating decision making and decision outcomes. Because value-based decisions cannot be judged as right or wrong, the framework defines an optimal decision as one that is informed, consistent with personal values, acted upon, and in which participants express satisfaction with decision-making (O'Connor, 1995). This framework was recently used to develop a DA for BRCA+ women who have never had breast cancer (Metcalfe et al., 2007).

Determination of Decision-Making Support Needs from Phase 1 Findings-The DA reflected the major decision topics identified in Phase 1 focus groups, risk-reducing mastectomy and risk-reducing salpingo-oophorectomy. Tamoxifen use was also included in the DA because of its potential for risk-reduction (King et al., 2001; Narod et al., 2000) and concern expressed about tamoxifen by women seen for risk assessment (MacDonald et al., 2002). Breast reconstruction and hysterectomy, while deemed important by Phase 1 participants, were not included in this initial DA because these decisions are typically made after risk-reducing mastectomy and risk-reducing salpingo-oophorectomy decisions and due to our concern that the DA would become too overwhelming.

Delivery Format-A web-based format for the DA was chosen for ease of use in various clinical and non-clinical settings, widespread dissemination, data collection, and capability for updating. SelectSurvey.NET 2.2.5 (Atomic Design, 2006) software was used to develop the DA, which could be accessed both inside and outside the City of Hope with data transmitted on a secure server.

Presentation of Risk and Risk-Reduction Options-The DA describes the risks for a new breast cancer and ovarian cancer and options for cancer screening and risk-reduction (NCCN, 2010). Breast screening options included mammography and breast MRI. Ovarian screening by CA125 and transvaginal ultrasound were described, noting their limitations 
(Modugno, 2003). Risk reduction strategies presented were risk-reducing mastectomy, riskreducing salpingo-oophorectomy, and tamoxifen.

Numerical Presentation of Risk Reduction-Iconic graphs were used to illustrate the relative risk reduction and potential side effects of risk-reducing mastectomy, risk-reducing salpingo-oophorectomy and tamoxifen (Figure 1) using the format described by Metcalfe et al (2007) which we modified to fit BRCA carriers with breast cancer (Barton et al., 2005; Eisen et al., 2005; Elit, Esplen, Butler, \& Narod, 2001; Fisher et al., 1998; Gronwald et al., 2006; Hartmann et al., 1999; Kramer et al., 2005; Metcalfe et al., 2004; Rebbeck et al., 2004; Rebbeck et al., 1999). Iconic graphs for risk prediction are the preferred method of the International Patient Decision Aids Standards Collaboration (IPDAS) (Elwyn et al., 2006), and were found acceptable to BRCA carriers (Metcalfe et al., 2007) and participants in one of the two Phase 1 focus groups (data not shown). The strength of available evidence for the risk reduction procedures were shown in the DA as depicted in Figure 1.

Values Ranking Exercise-The DA included a values ranking exercise for users to rate the personal importance of each potential benefit and harm associated with the three riskreduction options presented (Figure 2). Participants used a Likert scale ranging from 1 (matters a little) to 5 (matters a lot), with an additional option of 0 (does not matter at all), to provide insight into how personal values affect decision-making. The benefits and harms were derived from Phase 1 focus group findings. For example, a potential risk-reducing mastectomy harm was "Changes in Body Image" (Table II). Similar ranking exercises for risk-reducing salpingo-oophorectomy and tamoxifen were also included in the DA (not shown). Potential risk-reducing salpingo-oophorectomy benefits included: highest chance of preventing ovarian cancer, reduced risk of breast cancer if done before age 50, and avoids need for screening. Potential risk-reducing salpingo-oophorectomy harms included: surgical problems, menopausal symptoms, long-term menopausal effects, and loss of fertility. The exercises included an option to add and rank any additional benefits or harms of riskreducing mastectomy, risk-reducing salpingo-oophorectomy, or tamoxifen considered personally important.

Output Page-An output page from the DA reflects users' clarification of values and outlines the next steps to making a decision (Figure 3 ). This page provides each user with a take-home summary of her DA responses for each of the three decisions (risk-reducing mastectomy, risk-reducing salpingo-oophorectomy, and tamoxifen).

\section{Phase 2 Focus Groups}

After creating the DA, it was evaluated with nine, two-hour focus groups with a total of 47 participants which were conducted in 2007 and 2008 (Table I). We aimed to have two groups of 5-10 participants per category of $B R C A+$ patients with breast cancer, breast cancer advocates, genetics professionals, and oncology professionals. However, due to low attendance, a third patient focus group was held.

Participants-Recruitment procedures for Phase 2 patient focus groups were similar to Phase 1; 108 eligible women were identified. Advocates were defined as those actively involved in advancing the cause for women with breast cancer via peer support, cancer education, fundraising, community outreach, policy/legislative work, and/or grant review. Local advocate organizations, such as American Cancer Society and Wellness Community were contacted to recruit advocate participants. Genetics professionals were defined as genetic counselors, genetic nurses, and physicians who self-identified that genetics was their primary area of practice. Oncology professionals included both oncology nurses and physicians. The first genetics focus group consisted of colleagues in the Los Angeles area. 
The second genetics focus group and the two oncology focus groups consisted of volunteers from participants in the $2008 \mathrm{COH}$ Intensive Course in Cancer Genetics. All were practicing medical professionals throughout the U.S. with the exception of one genetics professional from Chile.

Procedures-Phase 2 Focus Groups followed the procedures used for Phase 1.

Additionally, each Phase 2 participant viewed the DA using a laptop computer. Participants were asked to consider the DA from the perspective of a $B R C A+$ patient with breast cancer who has recently received her genetic test results, and to evaluate the DA's usefulness, information content, emotional impact, aesthetics, timing, implementation, and output page.

Data Analysis-Data analysis procedures followed those used for Phase 1.

\section{RESULTS}

\section{Phase 1 Focus Groups}

Phase 1 focus groups were conducted with $B R C A+$ female breast cancer survivors to assess their decision-making needs related to risk-reduction. The women $(n=11)$ were mostly white, partnered, with a median age of 52 (Table I). All had completed childbearing with a median of 1 child (range 0-3). Fifty-five percent received their BRCA test results $\mathcal{\Omega}$ years ago and the remainder received their results $>2$ years ago. Eight participants had elected both risk-reducing mastectomy and risk-reducing salpingo-oophorectomy, one had elected risk-reducing mastectomy alone, one had elected risk-reducing salpingo-oophorectomy alone, and one had elected screening alone.

Participants expressed that the major decisions they faced upon learning their $B R C A$ status were related to risk reducing surgeries (risk-reducing mastectomy and risk-reducing salpingo-oophorectomy), breast reconstruction, hysterectomy, and ovarian cancer screening. Factors reported as most important regarding risk-reducing mastectomy and risk-reducing salpingo-oophorectomy decisions and the process by which these decisions were made are described in Table II. For example, a factor in the risk-reducing mastectomy decision was breast size: one woman saw this as an opportunity for breast reduction and another viewed it as enabling breast enlargement. Table II also includes the processes women described for risk-reducing mastectomy and risk-reducing salpingo-oophorectomy decisions. For example, some women who opted for risk-reducing mastectomy described how they considered the impact on their family in the process of decision making in several ways: by considering their family members' opinions, wanting to be alive for them, and wanting to serve as an example for daughters. Another process used was "weighing health versus appearance."

\section{Phase 2 Focus Groups}

Phase 2 focus groups were aimed at evaluating the utility and acceptability of the DA. Demographic and professional experience of the 47 participants are also shown in Table I. All patient participants and one advocate were $B R C A+$. The mean number of participants per focus groups was $5.2(+/-1.99$; range, 2 to 8$)$.

Values Ranking Exercise and Output Page-Feedback from all four participant categories was very positive regarding the clarification of values ranking exercise (Figure 2) and the output page (Figure 3). As summarized by an advocate: "I liked it specifically because it prioritizes; it makes you think 'How important is it to me to have a lack of intimacy?' or 'How important is it to me to reduce the fear of risk of recurrence?' and so by having those actual priorities on paper and then summarized at the end I think is extremely beneficial." 
Specific aspects of the values exercise were identified as being particularly useful. An oncology professional thought that the exercise would allow for a less emotionally intense values clarification process: "It helps a patient systematically analyze the different components that might play into the decision, and when you're forced to kind of rank them or qualify them...it takes away, perhaps, some of the emotion of it."

A patient felt that completing the exercise on the computer by herself helped avoid external pressures: "When it is in black and white in front of me and I am able to block out everybody else, what they want, what they think I should do and I can look at it and say what is the best thing step by step for me and then get a print out - that is huge."

Timing of the Decision Aid-The advocates and genetics professionals felt that the DA would be overwhelming for patients who had just received genetic test results. One advocate described the likely (or expected or typical) distressed emotional state of a woman who had just received $B R C A+$ genetic test results: "You just got this sort of bomb, as most people would consider it." A genetics professional expressed concern that a patient's comprehension might become impaired during results disclosure: "When you have a woman who gets a positive result, they don't pick up as much during that session. I think they can be so overwhelmed that it is really the last place I would put a decision aid like this." While some patients and oncology professionals expressed similar concerns, others felt that the DA would be most valuable immediately after the counseling session. A patient stated that the DA should be used "right afterwards...definitely." One oncologist said, "I feel compelled to give them that information the best I can, before they walk out." However, another oncology professional thought that the DA could be best used if provided at the time of pre-test counseling, which would allow for review of the DA prior to results disclosure. There was also concern that waiting too long would diminish the value of the DA, "....if they are answering it honestly and trying to use it as a decision aid, early is better than late."

Many focus group participants acknowledged that patients have different needs and that one model for use of the DA may not fit everyone. An oncology professional verbalized, "You might be able to tell that this is the kind of person who...is asking for everything that you've got or it might be the kind of person who is just overwhelmed with what you're telling them to begin with." A patient suggested that $B R C A+$ patients should be asked when they want to use the DA: "The patient will probably let you know if they are ready for it or not."

Decision Aid Setting-Use of a decision aid in the clinical setting was viewed as allowing a closer connection to the medical team which may be ideal for patients making surgical decisions. A patient wanted maximal support and guidance from health professionals by using the DA in a clinical setting: "Definitely I would want to have done it in an office so that if I had questions the professionals would have been there to answer." Another patient felt that she would take it more seriously in a clinic: "I think you would be more geared up for it, more open if it was, more serious about it if it was in an office than at home." A genetics professional suggested: "It is likely to be applied in the clinical setting because they are in the midst of making those decisions and trying to sort through all the data."

In contrast, an advocate expressed that at-home use may provide a calmer environment with the opportunity for family support: "If I am doing it in the comfort of my own home and with those around me who love me, then I am able to process that." One genetics professional stated that, "using the decision aid at home is much more comfortable." An oncology professional stated that the DA could be a useful home exercise in preparation for a medical appointment: "Before you see your surgeon or oncologist... here's a tool that you can do at home and then take with you." However, another oncology professional expressed 
concern that using the DA at home would not allow for clinical interpretation: "If you give this to them and it's the last time you see them and they either go to a website... and go do the exercise, are they really going to know how to self-interpret with what they've just done? And then they get to the end and go, 'Well that was interesting. Now what?'”

Numerical Presentation of Risk-Reduction-Most participants found the facial icons used to convey risk reduction (Figure 1) difficult to understand and voiced preference for graphs, numbers, or percentages. One patient associated sad faces with death and found them upsetting, several advocates found the faces offensive, and one advocate described them as "too cutesy." Additionally, most genetics and oncology professionals felt that the numerical risk presentation page contained too much information.

Similarly, the majority of participants agreed that the use of ribbons to illustrate the level of evidence associated with each risk reduction option was confusing. All four types of participant expressed that patients presume the evidence level of any data displayed is satisfactory. Further, given the lack of platinum-level evidence in this setting, they were concerned that DA users would find this discussion confusing or even disconcerting. Some suggested that a disclaimer and explanation regarding the strength of evidence would serve the same purpose and be easier to understand.

Risk Reduction Information Content-In general, participants in all focus groups agreed that the DA would facilitate processing of information needed by $B R C A+$ breast cancer patients who are making risk-reduction decisions. However, several areas for improvement were identified. Most participants wanted more information regarding the benefits, limitations, and risks of various risk-reducing options (i.e., whether temporary, permanent, or life-threatening), including the use of tamoxifen and oral contraceptives, as well as ovarian cancer screening. Information not presented in the DA, but deemed important, related to aromatase inhibitors, breast reconstruction, whether hysterectomy should be performed in conjunction with risk-reducing salpingo-oophorectomy and clarification of tamoxifen use for adjuvant therapy versus risk-reduction. Patients also desired an explanation of the menopausal quality of life changes that might be expected with use of tamoxifen or oophorectomy prior to menopause.

Regarding focus group differences, many advocates and genetics professionals consistently requested a higher level of detail, including specific percentages of outcomes related to the risk-reduction options and simpler definitions of terms. Yet other advocates were concerned that the amount of detail presented was already overwhelming. Oncologists felt that the information should be presented in simpler terms. Most participants thought that providing a paper copy of the entire DA would be useful to patients in reviewing the information for their own decision-making and in discussions with family members.

Tailoring-Focus group participants suggested variables that could be used to tailor the DA, including patient age, cancer characteristics (e.g., hormone receptor status), gene involved ( $B R C A 1$ versus $B R C A 2$ ), tamoxifen eligibility, reproductive milestones (completion of childbearing, menopausal status), educational level, relationship status, desired level of detail, and need for a support group.

\section{DISCUSSION}

This paper describes the development of a web-based DA to be used as an adjunct to genetic counseling for breast cancer patients who receive a $B R C A+$ genetic test result. This tool was designed for both women with a history of breast cancer who have a newly identified $B R C A$ 
+ mutation and women with a recent diagnosis of breast cancer who are using $B R C A+$ results to help make timely surgical decisions.

To our knowledge, this is the first DA developed specifically for $B R C A+$ women with a breast cancer that can be easily updated as new medical information becomes available. Two previous DAs created by van Roosmalen et al (van Roosmalen et al., 2004a) and Schwartz et al (Schwartz et al., 2009) were tailored by affected status to address the needs of women with breast cancer. However, neither of these DAs were created in an easily updatable format, and hence they do not include the most recent data, such as the use of breast MRI as a screening modality for the $B R C A+$ population, which is now standard-of-care. The Tiller et al. (2006) decision aid is tailored to the user's affected status, but the primary goal of their DA was to address ovarian cancer risk, not breast cancer risk. The Metcalfe et al. (2007) DA, while up-to-date, targets unaffected women only.

We report feedback on the DA provided by two sets of focus groups: $B R C A+$ breast cancer patients (Phase 1) and patients, advocates, and genetics and oncology professionals (Phase 2). In Phase 1, participants identified risk-reducing mastectomy, risk-reducing salpingooophorectomy, hysterectomy, breast reconstruction, and ovarian cancer screening as the key decisions they faced when making choices for reducing risk of a second primary cancer. These women also described their personal decision factors and the processes that guided their decision making. While previous studies of risk-reducing mastectomy decisions have similarly identified the role of anxiety and worry (Schwartz, Peshkin, Tercyak, Taylor, \& Valdimarsdottir, 2005), physician recommendations (Schwartz et al., 2004), opinions of family members (van Dijk, van Roosmalen, Otten, \& Stalmeier, 2008), screening effectiveness (Claes et al., 2005), body image (Claes et al., 2005), and surgery planned for breast treatment (Mai et al., 2008), we also found that planned mastectomy for breast cancer treatment and the impact of reconstruction on breast size (either larger or smaller) may be important decision-making factors. Importantly, we captured details of the process involved in making these decisions, including deliberations such as weighing health versus appearance, considering the example that would be set for a daughter, and/or delaying riskreducing salpingo-oophorectomy until attempting pregnancy. The few studies to date that describe the decision-making processes for risk-reducing mastectomy (Lloyd et al., 2000) or risk-reducing salpingo-oophorectomy (Hallowell, Jacobs, Richards, Mackay, \& Gore, 2001), found that these decisions are complex and dynamic, involving consideration of risk perception, individual and social factors, and family opinions. The present findings contribute to understanding how these decisions are made.

In the Phase 2 focus groups, we solicited detailed feedback regarding various features of the DA. Participants were generally positive about the role of this tool in a woman's riskreducing decision making, especially related to having a systematic process for identifying and weighing personal values and preferences. However, there was considerable discussion and debate about other features of the DA such as its informational content, presentation of risk-reducing options, and tailoring.

Differing views were also expressed about the ideal timing for using the DA. Generally, patients and oncology professionals were more practical in their feeling that patient decision-making should be closely coordinated with clinical care. In contrast, advocates and genetics providers were more protective of the patients' emotions and suggested personalized timing of the DA. When considering the best timing to introduce the DA, there is a delicate balance between too soon and possibly overwhelming a patient who has just received results, and waiting too long and therefore missing the opportunity to aid decisionmaking. Having immediate access to the DA would be especially important for women facing a recent diagnosis of breast cancer who typically need to make surgical treatment 
decisions within a short time-frame. van Roosmalen and colleagues (2004a) analyzed effects of timing of a brochure and videotape DA and found no differences in decision-making outcomes between women who had received the DA before genetic test results $(n=47)$ and after test results $(n=42)$. Schwartz et al. (2009) offered participation in a DA study one month after results disclosure. They found that $49 \%$ of their 214 participants had already reached a final management decision and ultimately did not benefit from the DA, whereas the undecided women did benefit. The ideal timing of such a DA may vary by each patient, depending on their specific circumstances and timeline for decision-making. Thus, timing is an issue requiring further investigation.

With respect to location of use, many advocates and providers suggested the DA be used athome, while patients unanimously preferred the clinical setting. In the Schwartz et al. (2009) randomized DA study, 35 of 100 participants randomized to use a CD ROM at home reported they never used the DA. While at-home use may be more convenient and facilitate dissemination, patient compliance and accessibility of the medical team are important concerns.

In the present study, participants perceived the two least favorable components of the DA to be the icons showing level of risk reduction and the ribbons showing the associated level of evidence (Figure 1). Although the IPDAS collaboration recommends using iconic graphs and including the level of evidence associated with each intervention, participants strongly disliked the icons and found the evidence information to be confusing. We previously examined ability to comprehend graphs in a population of women at high risk for breast cancer, the majority of which were BRCA+ (Brown et al., 2010). Participants in that study scored highest in graph comprehension when using an iconic graph. Ironically, performance and preference did not match; participants answered the fewest questions correctly when using the vertical bar graph but ranked this graph highest in personal preference.

The findings of Brown et al (2010) confirm previous studies wherein participants preferred simple bar graphs (Fortin, Hirota, Bond, O'Connor, \& Col, 2001), and preference was not always associated with comprehension(Ancker, Senathirajah, Kukafka, \& Starren, 2006). However, given the feedback obtained in the current study, and the effective use of bar graphs reported for other similar DAs (Schwartz et al., 2009; van Roosmalen et al., 2004b), we plan to use bar graphs to display cancer risk and risk reduction in revising the DA.

Similarly, given the strong feedback we received about displaying the level of evidence, we will use another format to simplify and de-emphasize this component.

Previous DAs were delivered in various formats including paper (Metcalfe et al., 2007,) CDROM (Schwartz et al., 2009), brochure and video (van Roosmalen, et al., 2004b), and a tailored booklet (Tiller et al., 2006), but none were available on the internet. We successfully implemented the DA on the web using a secure server without encountering significant logistical problems. Although a web-based format requires access to a computer, advantages include the ability to rapidly update medical information, disseminate the tool easily, and track compliance and usage patterns.

Participants in both focus group phases expressed the need for decision support related to breast reconstruction and preventive hysterectomy. Another common theme voiced by the participants was a need to tailor the DA to each patient's personal circumstances. In the next version we will tailor the DA to the participant's age, menopausal status, prior tamoxifen use, prior breast surgery, and oophorectomy history. This will allow us to add decision support such as breast reconstruction and risk reducing hysterectomy for women considering these options. 
The present study has some limitations. First, only a small proportion of eligible patients participated. Second, all of the patients resided in Southern California and may not be representative of other $B R C A$ mutation carriers with breast cancer. Third, the provider participants were colleagues or $\mathrm{COH}$ course attendees, and although geographically located across the U.S., they may not reflect the opinions of other genetics and oncology providers. Finally, Phase 2 participation may have been limited to those individuals willing to evaluate a computerized version of the DA.

\section{Future Directions}

The DA will be revised to incorporate focus group feedback regarding presentation of the risk-reducing options, tailoring the materials to each woman's breast cancer characteristics, utilizing individualized risk information (predictive model under development) (Burke et al., 2007) and addressing breast reconstructive options and hysterectomy. The revised DA will be pilot tested to assess logistical and psychosocial issues with clinic and at-home use, barriers to implementation, and usefulness in helping $B R C A+$ women with breast cancer develop an action plan. A future randomized controlled trial will further determine the effectiveness of the DA. We expect that the DA will ultimately prove to be an easily accessible and effective web-based tool to improve decision-making for $B R C A+$ women with breast cancer.

\section{Acknowledgments}

We thank the focus group participants and Janelle Hilario, Shawntel Payton, Judy Wong-Toh, and Tony Abell for their assistance with this project. Financial support for this study was provided by a grant from the Susan G. Komen Breast Cancer Foundation (Grant \# BCTR0600463) and in part by a General Clinical Research Center grant from NIH (M01 RR00043) and Cancer Center Support Grant (NCI-P20 CA 33572) awarded to the City of Hope National Medical Center, Duarte, California.

\section{REFERENCES}

1. Ancker JS, Senathirajah Y, Kukafka R, Starren JB. Design Features of Graphs in Health Risk Communication: A Systematic Review. Journal of the American Medical Informatics Association. 2006; 13(6):608-618. [PubMed: 16929039]

2. Antoniou A, Pharoah PD, Narod S, Risch HA, Eyfjord JE, Hopper JL, et al. Average Risks of Breast and Ovarian Cancer Associated with Brca1 or Brca2 Mutations Detected in Case Series Unselected for Family History: A Combined Analysis of 22 Studies. Am J Hum Genet. 2003; 72(5):1117-1130. doi: 10.1086/375033. [PubMed: 12677558]

3. Atomic Design, L. [Retrieved March 2007] Selectsurvey.Net 2.3.4. 2006. ClassApps.comwww.selectsurvey.net

4. Barton MB, West CN, Liu IL, Harris EL, Rolnick SJ, Elmore JG, et al. Complications Following Bilateral Prophylactic Mastectomy. Journal of the National Cancer Institute Monographs. 2005; (35):61-66. [PubMed: 16287887]

5. Braun V, Clarke V. Using Thematic Analysis in Psychology. Qualitative Research in Psychology. 2006; 3(2):77-101.

6. Brown SM, Culver JO, Osann KE, MacDonald DJ, Sand S, Thornton AA, et al. Health Literacy, Numeracy, and Interpretation of Graphical Breast Cancer Risk Estimates. Patient Education and Counseling. 2010 Epub ahead of print, June 42010.

7. Burke, H.; Hoang, A.; Metcalfe, K.; Culver, J.; MacDonald, D.; Grant, M., et al. Individualized Risk Predictions to Estimate the Clinical Benefit of Risk Reduction Mastectomy (Rrm) and Oophorectomy (Rrso). Brca Carriers with Breast Cancer 57th Annual American Society of Human Genetics Meeting; San Diego, CA. 2007. p. 103Retrieved from http://www.ashg.org/genetics/ashg/ annmeet/2007/ 
8. Claes E, Evers-Kiebooms G, Decruyenaere M, Denayer L, Boogaerts A, Philippe K, et al. Surveillance Behavior and Prophylactic Surgery after Predictive Testing for Hereditary Breast/ Ovarian Cancer. Behavioral Medicine. 2005; 31(3):93-105. [PubMed: 16252621]

9. Crotser CB, Boehmke M. Survivorship Considerations in Adults with Hereditary Breast and Ovarian Cancer Syndrome: State of the Science. J Cancer Surviv. 2009; 3(1):21-42. [PubMed: 19165605]

10. Croyle RT, Smith KR, Botkin JR, Baty B, Nash J. Psychological Responses to Brca1 Mutation Testing: Preliminary Findings. Health Psychol. 1997; 16(1):63-72. [PubMed: 9028816]

11. Domchek SM, Friebel TM, Singer CF, Evans DG, Lynch HT, Isaacs C, et al. Association of RiskReducing Surgery in Brca1 or Brca2 Mutation Carriers with Cancer Risk and Mortality. JAMA. 2010; 304(9):967-975. doi: 10.1001/jama.2010.1237. [PubMed: 20810374]

12. Eisen A, Lubinski J, Klijn J, Moller P, Lynch HT, Offit K, et al. Breast Cancer Risk Following Bilateral Oophorectomy in Brca1 and Brca2 Mutation Carriers: An International Case-Control Study. J Clin Oncol. 2005; 23(30):7491-7496. [PubMed: 16234515]

13. Elit L, Esplen MJ, Butler K, Narod S. Quality of Life and Psychosexual Adjustment after Prophylactic Oophorectomy for a Family History of Ovarian Cancer. Fam Cancer. 2001; 1(3-4): 149-156. [PubMed: 14574171]

14. Elwyn G, O'Connor A, Stacey D, Volk R, Edwards A, Coulter A, et al. Developing a Quality Criteria Framework for Patient Decision Aids: Online International Delphi Consensus Process. BMJ. 2006; 333(7565):417. [PubMed: 16908462]

15. Ferrell BR, Grant MM, Funk B, Otis-Green S, Garcia N. Quality of Life in Breast Cancer Survivors as Identified by Focus Groups. Psycho-Oncology. 1997; 6:13-25. [PubMed: 9126712]

16. Fisher B, Costantino JP, Wickerham DL, Redmond CK, Kavanah M, Cronin WM, et al. Tamoxifen for Prevention of Breast Cancer: Report of the National Surgical Adjuvant Breast and Bowel Project P-1 Study. J Natl Cancer Inst. 1998; 90(18):1371-1388. [PubMed: 9747868]

17. Ford D, Easton DF, Bishop DT, Narod SA, Goldgar DE, Consortium TBCL. Risks of Cancer in Brca1-Mutation Carriers. Lancet. 1994; 343:692-695. [PubMed: 7907678]

18. Ford D, Easton DF, Stratton M, Narod S, Goldgar D, Devilee P, et al. Genetic Heterogeneity and Penetrance Analysis of the Brca1 and Brca2 Genes in Breast Cancer Families. Am J Hum Genet. 1998; 62:676-689. doi: 10.1086/301749. [PubMed: 9497246]

19. Fortin JM, Hirota LK, Bond BE, O'Connor AM, Col NF. Identifying Patient Preferences for Communicating Risk Estimates: A Descriptive Pilot Study. BMC Med Inform Decis Mak. 2001; 1(1):2. [PubMed: 11545684]

20. Gronwald J, Tung N, Foulkes WD, Offit K, Gershoni R, Daly M, et al. Tamoxifen and Contralateral Breast Cancer in Brca1 and Brca2 Carriers: An Update. Int J Cancer. 2006; 118(9): 2281-2284. [PubMed: 16331614]

21. Hallowell N, Jacobs I, Richards M, Mackay J, Gore M. Surveillance or Surgery? A Description of the Factors That Influence High Risk Premenopausal Women's Decisions About Prophylactic Oophorectomy. Journal of Medical Genetics. 2001; 38(10):683-691. [PubMed: 11594337]

22. Hartmann LC, Schaid DJ, Woods JE, Crotty TP, Myers JL, Arnold PG, et al. Efficacy of Bilateral Prophylactic Mastectomy in Women with a Family History of Breast Cancer. New England Journal of Medicine. 1999; 340(2):77-84. [PubMed: 9887158]

23. Hooker GW, Leventhal KG, Demarco T, Peshkin BN, Finch C, Wahl E, et al. Longitudinal Changes in Patient Distress Following Interactive Decision Aid Use among Brca1/2 Carriers: A Randomized Trial. Medical Decision Making. 2010 doi: 10.1177/0272989X10381283.

24. Hsieh HF, Shannon SE. Three Approaches to Qualitative Content Analysis. Qualitative Health Research. 2005; 15(9):1277-1288. [PubMed: 16204405]

25. Kauff ND, Satagopan JM, Robson ME, Scheuer L, Hensley M, Hudis CA, et al. Risk-Reducing Salpingo-Oophorectomy in Women with a Brca1 or Brca2 Mutation. New England Journal of Medicine. 2002; 346(21):1609-1615. [PubMed: 12023992]

26. King M-C, Wieand S, Hale K, Lee M, Walsh T, Owens K, et al. Tamoxifen and Breast Cancer Incidence among Women with Inherited Mutations in Brca1 and Brca2: National Surgical Adjuvant Breast and Bowel Project (Nsabp-P1) Breast Cancer Prevention Trial. JAMA. 2001; 286(18):2251-2256. [PubMed: 11710890] 
27. Kramer JL, Velazquez IA, Chen BE, Rosenberg PS, Struewing JP, Greene MH. Prophylactic Oophorectomy Reduces Breast Cancer Penetrance During Prospective, Long-Term Follow-up of Brca1 Mutation Carriers. J Clin Oncol. 2005; 23(34):8629-8635. [PubMed: 16314625]

28. Krueger, RA.; Casey, MA. Focus Groups: A Practical Guide for Applied Research. Vol. edition. Thousand Oaks, Calif.: 2000.

29. Lloyd SM, Watson M, Oaker G, Sacks N, Querci della Rovere U, Gui G. Understanding the Experience of Prophylactic Mastectomy: A Qualitative Study of Ten Women. Psycho-Oncology. 2000; 9(6):473-485. [PubMed: 11180582]

30. MacDonald DJ, Choi J, Ferrell B, Sand S, McCaffrey S, Blazer KR, et al. Concerns of Women Presenting to a Comprehensive Cancer Center for Genetic Cancer Risk Assessment. Journal of Medical Genetics. 2002; 39(7):526-530. [PubMed: 12114489]

31. Mai PL, Lagos VI, Palomares MR, Weitzel JN. Contralateral Risk-Reducing Mastectomy in Young Breast Cancer Patients with and without Genetic Cancer Risk Assessment. Annals of Surgical Oncology. 2008; 15(12):3415-3421. doi: 10.1245/s10434-008-0160-3. [PubMed: 18836779]

32. McDonnell SK, Schaid DJ, Myers JL, Grant CS, Donohue JH, Woods JE, et al. Efficacy of Contralateral Prophylactic Mastectomy in Women with a Personal and Family History of Breast Cancer. J Clin Oncol. 2001; 19:3938-3943. [PubMed: 11579114]

33. Metcalfe K, Lynch HT, Ghadirian P, Tung N, Olivotto I, Warner E, et al. Contralateral Breast Cancer in Brca1 and Brca2 Mutation Carriers. J Clin Oncol. 2004; 22(12):2328-2335. doi: 10.1200/JCO.2004.04.033. [PubMed: 15197194]

34. Metcalfe KA, Poll A, O'Connor A, Gershman S, Armel S, Finch A, et al. Development and Testing of a Decision Aid for Breast Cancer Prevention for Women with a Brca1 or Brca2 Mutation. Clinical Genetics. 2007; 72(3):208-217. [PubMed: 17718858]

35. Modugno F. Ovarian Cancer and High-Risk Women-Implications for Prevention, Screening, and Early Detection. Gynecol Oncol. 2003; 91(1):15-31. [PubMed: 14529658]

36. Muhr, T. Atlas.Ti. Version 5.0. Berlin: 2005. Retrieved from www.atlasti.com

37. Narod SA, Brunet JS, Ghadirian P, Robson M, Heimdal K, Neuhausen SL, et al. Tamoxifen and Risk of Contralateral Breast Cancer in Brca1 and Brca2 Mutation Carriers: A Case-Control Study. Lancet. 2000; 356:1876-1881. doi: 10.1016/S0140-6736(00)03258-X. [PubMed: 11130383]

38. NCCN. Nccn Practice Guidelines V.1.2010: Genetic/Familial High-Risk Assessment: Breast and Ovarian. NCCN Practice Guidelines. 2010; 8(5):562-594. Retrieved from http:// www.ncbi.nlm.nih.gov/entrez/query.fcgi? $\mathrm{cmd}=$ Retrieve $\& \mathrm{db}=$ PubMed\&dopt=Citation\&list_uids=20495085.

39. Neuendorf, K. The Content Analysis Guidebook. CA Sage; Thousand Oaks: 2002.

40. O’Brien MA, Whelan TJ, Villasis-Keever M, Gafni A, Charles C, Roberts R, et al. Are CancerRelated Decision Aids Effective? A Systematic Review and Meta-Analysis. J Clin Oncol. 2009; 27(6):974-985. doi: 10.1200/jco.2007.16.0101. [PubMed: 19124808]

41. O'Connor AM. Validation of a Decisional Conflict Scale. Medical Decision Making. 1995; 15(1): 25-30. [PubMed: 7898294]

42. O’Connor AM, Fiset V, DeGrasse C, Graham ID, Evans W, Stacey D, et al. Decision Aids for Patients Considering Options Affecting Cancer Outcomes: Evidence of Efficacy and Policy Implications. Journal of the National Cancer Institute Monographs. 1999; (25):67-80. [PubMed: 10854460]

43. O’Connor, AM.; Stacey, D.; Entwistle, V.; Llewellyn-Thomas, H.; Rovner, D.; Holmes-Rovner, M., et al. [Retrieved 11 May 2005] Decision Aids for People Facing Health Treatment or Screening Decisions. Cochrane Database of Systematic Reviews Issue 1. 2004. from http:// 204.187.39.28/cochsystem.html

44. O'Connor AM, Tugwell P, Wells GA, Elmslie T, Jolly E, Hollingworth G, et al. Randomized Trial of a Portable, Self-Administered Decision Aid for Postmenopausal Women Considering LongTerm Preventive Hormone Therapy. Medical Decision Making. 1998; 18(3):295-303. [PubMed: 9679994]

45. Rebbeck TR, Friebel T, Lynch HT, Neuhausen SL, van't Veer L, Garber JE, et al. Bilateral Prophylactic Mastectomy Reduces Breast Cancer Risk in Brcal and Brca2 Mutation Carriers: The 
Prose Study Group. J Clin Oncol. 2004; 22(6):1055-1062. doi: 10.1200/jco.2004.04.188. [PubMed: 14981104]

46. Rebbeck TR, Kauff ND, Domchek SM. Meta-Analysis of Risk Reduction Estimates Associated with Risk-Reducing Salpingo-Oophorectomy in Brca1 or Brca2 Mutation Carriers. J Natl Cancer Inst. 2009; 101(2):80-87. doi: 10.1093/jnci/djn442. [PubMed: 19141781]

47. Rebbeck TR, Levin AM, Eisen A, Snyder C, Watson P, Cannon-Albright L, et al. Breast Cancer Risk after Bilateral Prophylactic Oophorectomy in Brca1 Mutation Carriers. J Natl Cancer Inst. 1999; 91:1475-1479. [PubMed: 10469748]

48. Rebbeck TR, Lynch HT, Neuhausen SL, Narod SA, Van't Veer L, Garber JE, et al. Prophylactic Oophorectomy in Carriers of Brca1 or Brca2 Mutations. New England Journal of Medicine. 2002; 346(21):1616-1622. [PubMed: 12023993]

49. Schwartz MD, Lerman C, Brogan B, Peshkin BN, Hughes Halbert C, DeMarco T, et al. Impact of Brca1/Brca2 Counseling and Testing on Newly Diagnosed Breast Cancer Patients. J Clin Oncol. 2004; 22(10):1823-1829. [PubMed: 15067026]

50. Schwartz MD, Peshkin BN, Tercyak KP, Taylor KL, Valdimarsdottir H. Decision Making and Decision Support for Hereditary Breast-Ovarian Cancer Susceptibility. [Special Issue]. Health Psychol. 2005; 24(4 Suppl):S78-S84. doi: 1930-7810. [PubMed: 16045423]

51. Schwartz MD, Valdimarsdottir HB, DeMarco TA, Peshkin BN, Lawrence W, Rispoli J, et al. Randomized Trial of a Decision Aid for Brca1/Brca2 Mutation Carriers: Impact on Measures of Decision Making and Satisfaction. Health Psychol. 2009; 28(1):11-19. doi: 10.1037/a0013147. [PubMed: 19210013]

52. Struewing JP, Hartge P, Wacholder S, Baker SM, Berlin M, McAdams M, et al. The Risk of Cancer Associated with Specific Mutations of Brcal and Brca2 among Ashkenazi Jews. New England Journal of Medicine. 1997; 336(20):1401-1408. doi: 10.1056/NEJM199705153362001. [PubMed: 9145676]

53. Tiller K, Meiser B, Gaff C, Kirk J, Dudding T, Phillips KA, et al. A Randomized Controlled Trial of a Decision Aid for Women at Increased Risk of Ovarian Cancer. Medical Decision Making. 2006; 26(4):360-372. [PubMed: 16855125]

54. van Dijk S, van Roosmalen MS, Otten W, Stalmeier PFM. Decision Making Regarding Prophylactic Mastectomy: Stability of Preferences and the Impact of Anticipated Feelings of Regret. J Clin Oncol. 2008; 26(14):2358-2363. doi: 10.1200/jco.2006.10.5494. [PubMed: 18467728]

55. van Roosmalen MS, Stalmeier PF, Verhoef LC, Hoekstra-Weebers JE, Oosterwijk JC, Hoogerbrugge N, et al. Randomised Trial of a Decision Aid and Its Timing for Women Being Tested for a Brca1/2 Mutation. British Journal of Cancer. 2004a; 90(2):333-342. [PubMed: 14735173]

56. van Roosmalen MS, Stalmeier PF, Verhoef LC, Hoekstra-Weebers JE, Oosterwijk JC, Hoogerbrugge N, et al. Randomized Trial of a Shared Decision-Making Intervention Consisting of Trade-Offs and Individualized Treatment Information for Brca1/2 Mutation Carriers. J Clin Oncol. 2004b; 22(16):3293-3301. [PubMed: 15310772]

57. Waljee JF, Rogers MA, Alderman AK. Decision Aids and Breast Cancer: Do They Influence Choice for Surgery and Knowledge of Treatment Options? J Clin Oncol. 2007; 25(9):1067-1073. [PubMed: 17369570]

58. Watson M, Foster C, Eeles R, Eccles D, Ashley S, Davidson R, et al. Psychosocial Impact of Breast/Ovarian (Brca1/2) Cancer-Predictive Genetic Testing in a Uk Multi-Centre Clinical Cohort. British Journal of Cancer. 2004; 91(10):1787-1794. [PubMed: 15505627]

59. Weitzel JN, McCaffrey SM, Nedelcu R, MacDonald DJ, Blazer KR, Cullinane CA. Effect of Genetic Cancer Risk Assessment on Surgical Decisions at Breast Cancer Diagnosis. Archives of Surgery. 2003; 138(12):1323-1329. [PubMed: 14662532]

60. Whelan T, Levine M, Willan A, Gafni A, Sanders K, Mirsky D, et al. Effect of a Decision Aid on Knowledge and Treatment Decision Making for Breast Cancer Surgery: A Randomized Trial. JAMA. 2004; 292(4):435-441. [PubMed: 15280341] 


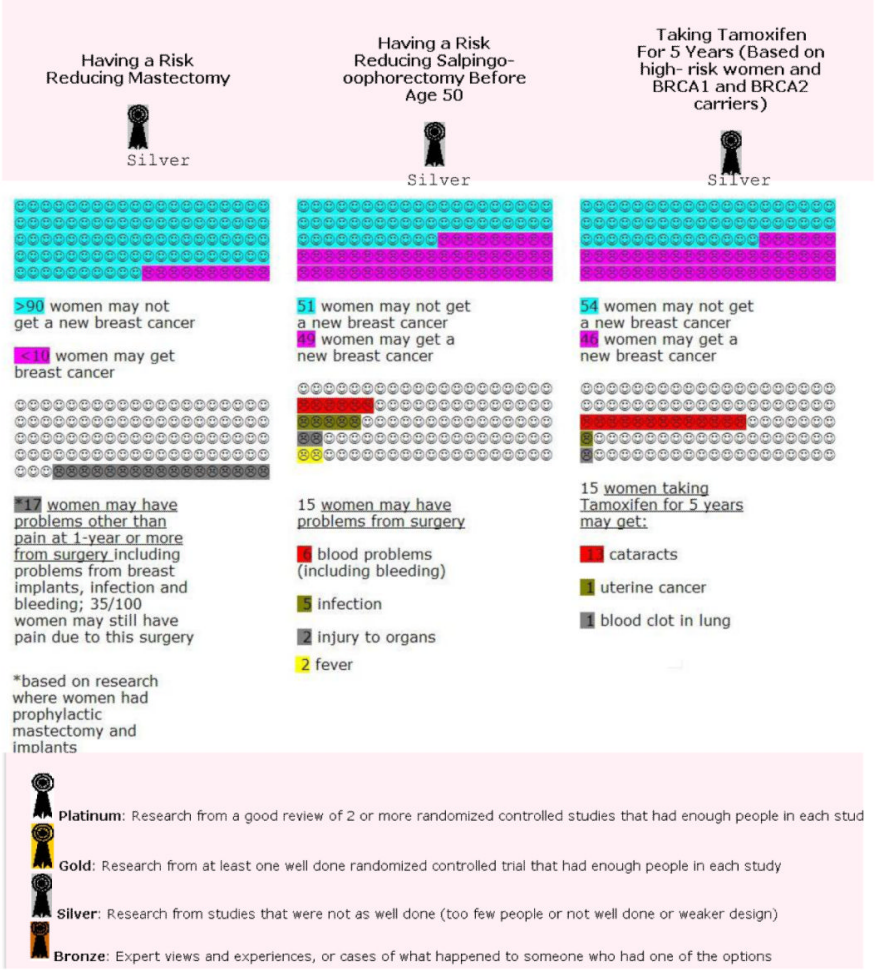

Figure 1. Numerical Presentation of Risk Reduction

In the decision aid the relative risk reduction and potential side effects of mastectomy, salpingo-oophorectomy and tamoxifen for risk-reduction, and the strength of evidence for these findings, are illustrated with iconic graphs. 


\section{BENEFITS}

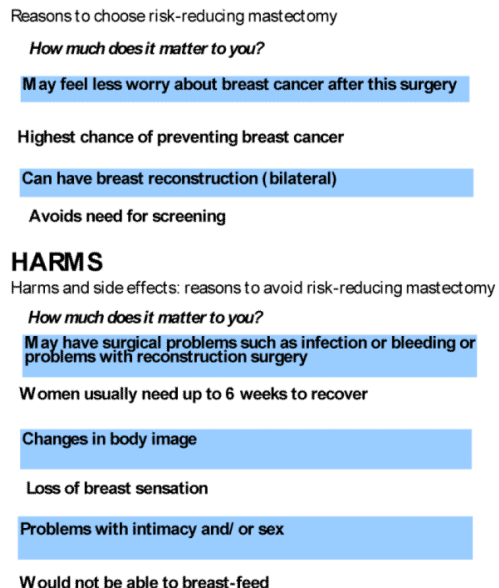

\begin{tabular}{cccccc}
$\begin{array}{c}\text { Does Not } \\
\text { Matter } \\
\text { at all }\end{array}$ & $\begin{array}{c}\text { Matters } \\
\text { a little }\end{array}$ & & & & \multicolumn{1}{c}{$\begin{array}{c}\text { Matters } \\
\text { a lot }\end{array}$} \\
0 & 1 & 2 & 3 & 4 & 5 \\
\hline 0 & 1 & 2 & 3 & 4 & 5 \\
\hline 0 & 1 & 2 & 3 & 4 & 5 \\
\hline 0 & 1 & 2 & 3 & 4 & 5 \\
\hline $\begin{array}{c}\text { Does Not } \\
\text { Matter } \\
\text { at all }\end{array}$ & $\begin{array}{c}\text { Matters } \\
\text { a little }\end{array}$ & & & & \\
\hline 0 & 1 & 2 & 3 & 4 & 5 \\
\hline 0 & 1 & 2 & 3 & 4 & 5 \\
\hline 0 & 1 & 2 & 3 & 4 & 5 \\
\hline 0 & 1 & 2 & 3 & 4 & 5 \\
\hline 0 & 1 & 2 & 3 & 4 & 5 \\
\hline 0 & 1 & 2 & 3 & 4 & 5
\end{tabular}

Figure 2. Values Clarification Exercise: Benefits and Harms of Risk-Reducing Mastectomy Decision aid users complete a values clarification exercise in which they rank how much each potential benefit and harm of risk-reducing mastectomy matters to them. 


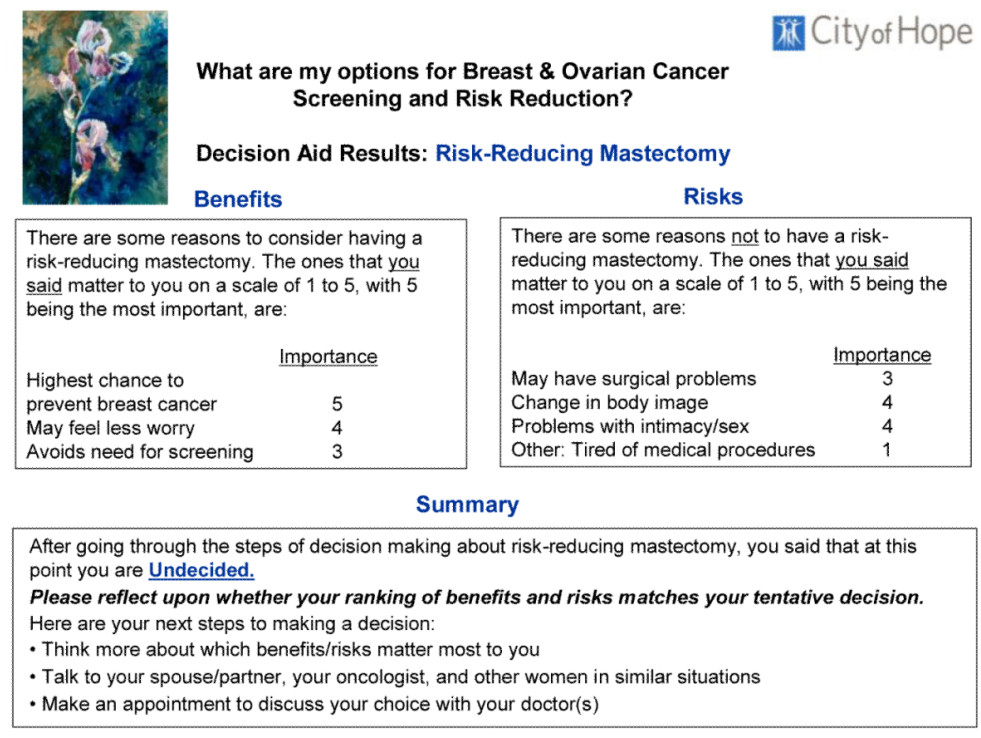

Figure 3. Sample Output Page for Risk-Reducing Mastectomy

Individualized responses to questions in the decision aid about risk-reducing mastectomy are reflected in an output page for participants to take home. 


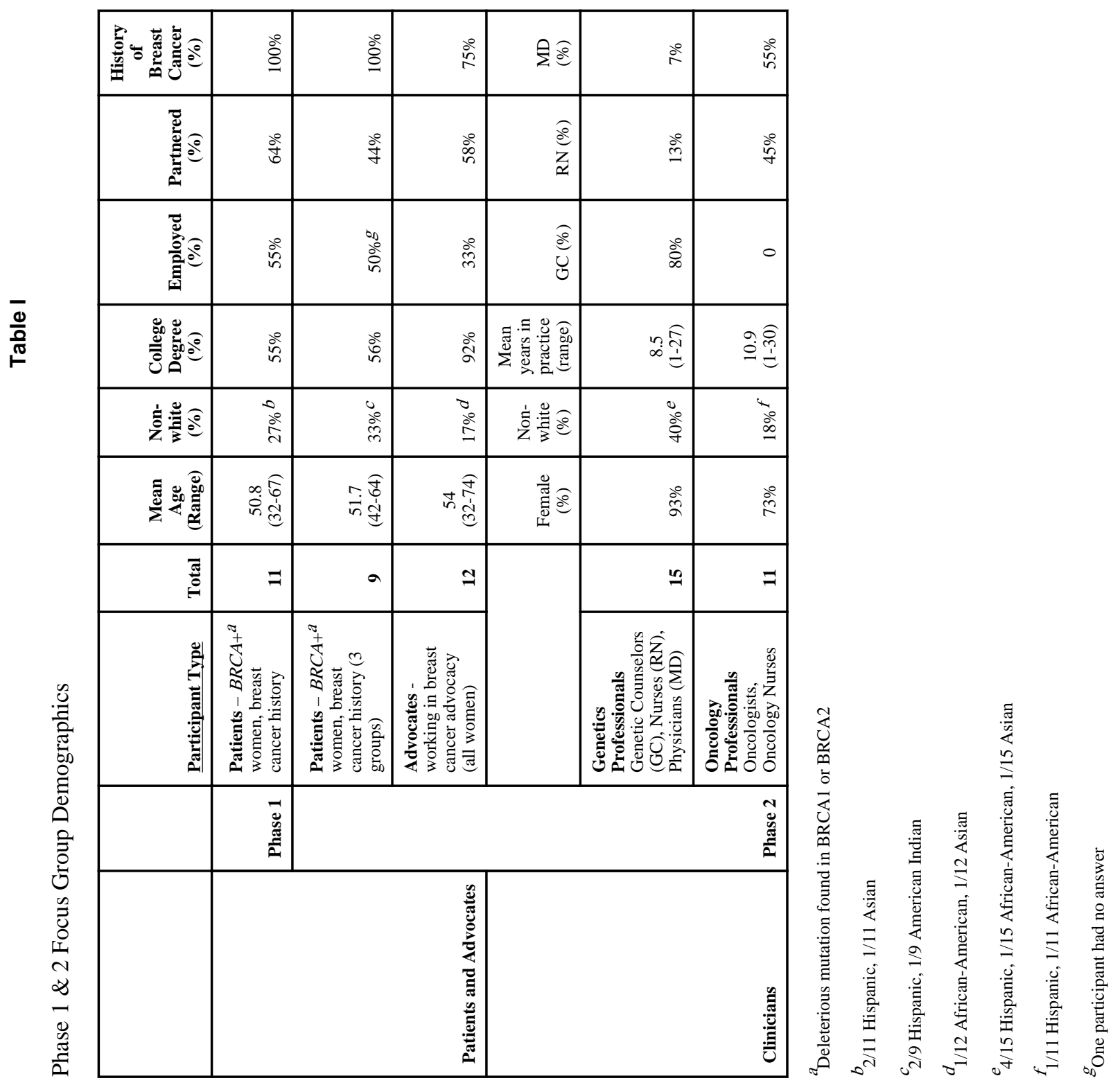

J Genet Couns. Author manuscript; available in PMC 2012 December 27. 
Table II

Phase 1 Focus Group Results: Decision Making Factors and Process Regarding Risk Reducing Mastectomy and Oophorectomy $(n=11)$

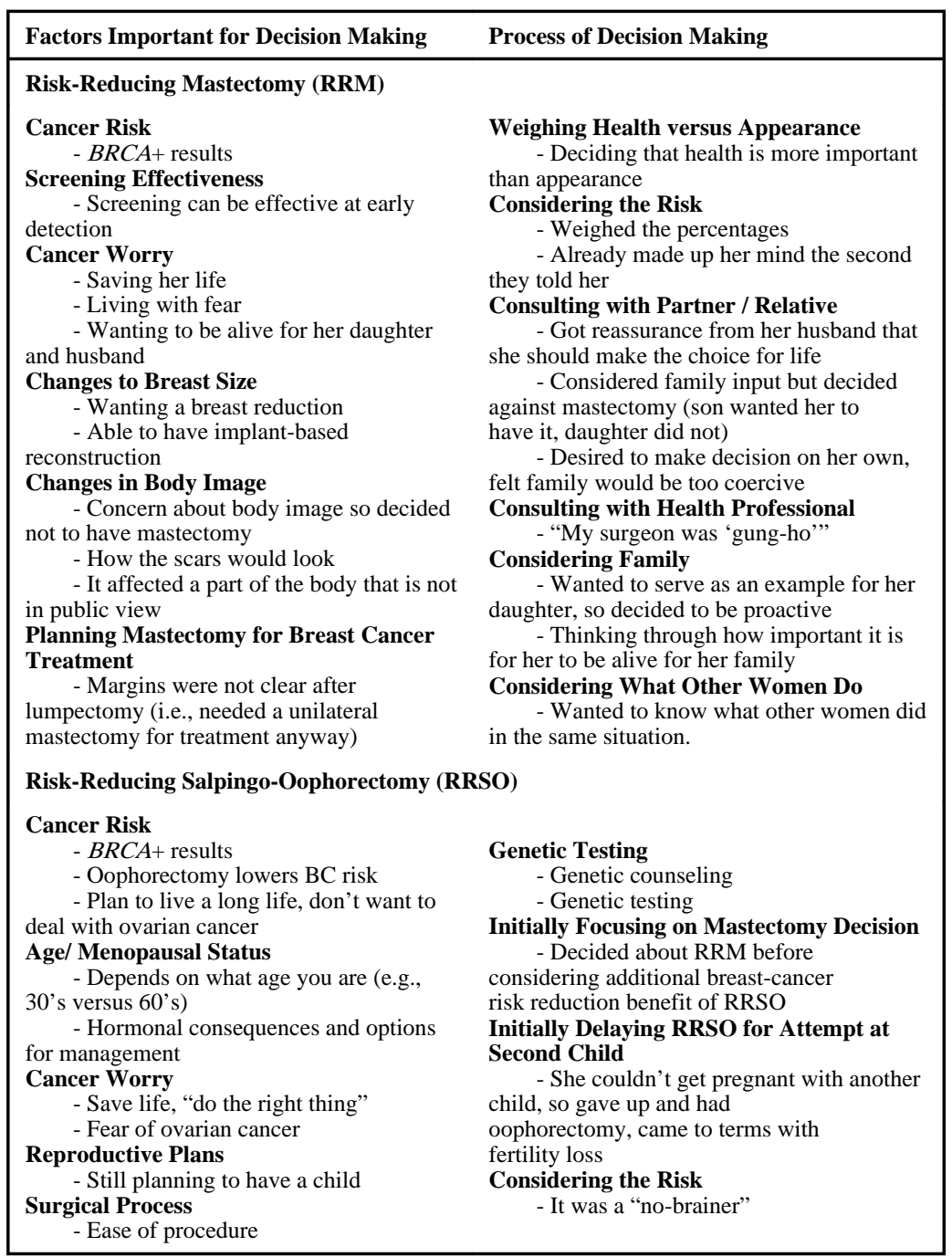

